\title{
Lidil
}

Revue de linguistique et de didactique des langues

\section{Relations esthétique, éthique et émotionnelle au texte littéraire. Deux textes contrastés au fil des niveaux scolaires}

Esthetic, Ethic and Emotional Relationships toward the Literary Text. Two

Contrasted Text in Function of School Levels

Chloé Gabathuler et Bernard Schneuwly

\section{OpenEdition}

Journals

Édition électronique

URL : http://journals.openedition.org/lidil/3499

DOI : 10.4000/lidil.3499

ISSN : $1960-6052$

Éditeur

UGA Éditions/Université Grenoble Alpes

Édition imprimée

Date de publication : 25 mai 2014

Pagination : 153-176

ISBN : 978-2-84310-272-1

ISSN : $1146-6480$

\section{Référence électronique}

Chloé Gabathuler et Bernard Schneuwly, « Relations esthétique, éthique et émotionnelle au texte littéraire. Deux textes contrastés au fil des niveaux scolaires », Lidil [En ligne], 49 | 2014, mis en ligne le 25 novembre 2015, consulté le 02 mai 2019. URL : http://journals.openedition.org/lidil/3499; DOI : 10.4000/lidil.3499 


\title{
Relations esthétique, éthique et émotionnelle au texte littéraire. Deux textes contrastés au fil des niveaux scolaires
}

\author{
Chloé Gabathuler et Bernard Schneuwly*
}

\begin{abstract}
Résumé
Nous nous intéressons aux relations créées entre élèves et œuvre littéraire par l'introduction d'un texte dans la classe et par les dispositifs mis en place par l'enseignant. Ces relations peuvent être décrites en fonction de trois dimensions profondément corrélées l'une à l'autre : les relations esthétique, éthique et émotionnelle. Afin de mieux comprendre comment s'instaurent ces trois relations en classe et les évaluations qui en découlent, mais aussi comment elles participent à la formulation d'interprétations, nous analyserons comment deux textes contrastés - l'un fortement didactisé et appartenant à la vulgate scolaire (Le loup et l'agneau de Jean de La Fontaine), l'autre issu de l'extrême contemporain et n'ayant fait l'objet d'aucune didactisation (La négresse et le chef des avalanches de Jean-Marc Lovay) - sont abordés dans des classes de primaire (10-11 ans), de secondaire I (13-14 ans) et de secondaire II (16-17 ans). Nous chercherons à comparer ainsi l'impact du texte et du niveau scolaire sur l'élaboration et la formulation de jugements esthétiques, éthiques et émotionnels ainsi que sur la construction d'interprétations.
\end{abstract}

\begin{abstract}
This paper analyzes the relationships created between students and literary work in function of a text introduced in the classroom and of the means used by a teacher for teaching. These relationships can be described following three deeply correlated dimensions: esthetic, ethic and emotional. In order to understand how these three types of relationships are created in the classroom and which evaluations are built
\end{abstract}

* Groupe de recherche pour l'analyse du français enseignée (GRAFE), Université de Genève. 
we have organized the following experiment: ten teachers in primary (10-11 years), secondary (13-14 years) and upper secondary level (1617 years) were asked to approach two contrasted texts in class: one is strongly "didactized" and belongs to the school canon since ever (Le loup et l'agneau by Jean de La Fontaine); the other is a contemporary text that has never been used in school and is no "didactised" at all (La négresse et le chef des avalanches by Jean-Marc Lovay). We analyze and compare the effect of the text and of the school level on the elaboration and formulation of esthetic, ethic and emotional judgments and on the construction of interpretations.

\section{Introduction}

Lorsqu'un texte littéraire est introduit en classe, des relations se créent entre ce texte et les élèves. Ces relations sont produites d'une part par l'enseignant et son action didactique, par le texte lui-même et ses spécificités et par les attentes des élèves vis-à-vis du texte littéraire en contexte d'enseignement. Cet article se penche tout particulièrement sur ces relations dans des classes du primaire (10-11 ans), du secondaire I (13-14 ans) et du secondaire II (16-17 ans) relativement à deux textes littéraires contrastés Le loup et l'agneau de Jean de La Fontaine et La négresse et le chef des avalanches de Jean-Marc Lovay. À travers l'analyse de discours élaborés tant par les enseignants que par les élèves sur ces deux textes littéraires, nous cherchons à définir la nature de ces relations. Notre regard porte plus particulièrement sur les jugements formulés vis-à-vis des deux textes. Avons-nous affaire à une relation esthétique à l'œuvre littéraire? Le cas échéant, quelles sont les caractéristiques de cette relation esthétique «scolaire» et dans quelle mesure se distingue-t-elle des approches de référence en la matière ${ }^{1}$ ? Nous interrogeons également la place occupée par la question des valeurs et de la morale dans l'établissement de relations à l'œuvre littéraire en classe. Est-ce que, par exemple, les finalités humanistes traditionnellement conférées à l'enseignement de la littérature transparaissent dans les discours sur les œuvres? Par ailleurs, nous nous intéressons à la place occupée par la dimension émotionnelle ou psychoaffective dans l'appréhension des deux textes. Quelle place est accordée au (dé)

1. Nous nous référons essentiellement aux domaines de l'esthétique philosophique et aux études littéraires. 
plaisir lors de leur lecture? Dans quelle mesure la relation au texte est-elle perçue comme surprenante, suscitant l'intérêt et l'imaginaire, ou comme le lieu d'identification? Après un développement théorique des trois relations aux textes, nous analysons quantitativement l'ensemble des jugements repérés dans les discours, puis montrons quelques exemples significatifs de co-constructions de jugements.

\section{Quelques jalons théoriques}

Afin de mieux saisir la nature des relations à l'œuvre littéraire ayant cours en classe, revenons tout d'abord aux disciplines de référence afin de définir à priori ce que nous entendons par relations esthétique, éthique et émotionnelle.

\subsection{Relation esthétique et jugement}

À la suite d'autres théoriciens avant nous, d'un point de vue théorique et en fonction de nos données empiriques, nous partons de Kant pour qui l'appréciation esthétique, bien que fondamentalement subjective, porte en elle une «prétention à l'universalité» (Kant, 1790/2011, p. 18). Ce que nous comprenons à travers cette «prétention», c'est la dimension intersubjective du jugement. Kant associe «un sentiment subjectif à la visée d'un partage nécessaire et universel», juger devenant ainsi «juger pour autrui» (Uzel, 2004). Dans sa Critique de la faculté de juger, Kant distingue le jugement de gout d'autres jugements par le fait d'être sans concept et désintéressé. Contrairement au jugement moral qui, se rapportant à un concept, vise un intérêt ou une «utilité», le jugement de gout est contemplatif, «il est son propre but» (Sapiro, 2010, p. 14). Cette question de l'évaluation esthétique et du jugement, pourtant centrale chez Kant, a été mise de côté par la tradition spéculative de la philosophie continentale ${ }^{2}$. Ce n'est que dans les années 1990 que certains auteurs tels que Genette, Schaeffer ou Rochlitz l'ont remise sur le devant de la scène (Vouilloux, 2011). Genette (1996) et Schaeffer (1997) reprennent à leur compte l'approche solipsiste du jugement et interprètent la «prétention à l'universalité» kantienne comme une illusion qui pousserait le sujet esthétique à objectiver son gout en

2. Ce qui n'est pas le cas de la philosophie analytique, voir à ce propos Vouilloux (2011). 
une propriété de la chose. Ce faisant, ils relèguent au second plan la dimension intersubjective du jugement pressentie par Kant et évacuent ainsi toute possibilité d'argumentation. La relation esthétique apparait comme une affaire personnelle et privée difficilement communicable. Or, il nous semble qu'une telle vision ne rend pas réellement compte de pratiques sociales courantes relatives aux textes littéraires ${ }^{3}$ et, qui plus est, semble bien éloignée de la classe de français. Nous rejoignons à ce propos la position d'un esthéticien comme Rochlitz (1998) qui, partant de la prémisse kantienne, affirme que les œuvres possèdent une signification publique, qu'elles sont des symboles en quête de reconnaissance et à propos desquelles l'argumentation est bel et bien possible. Le jugement serait à la base de toute activité esthétique, la plus profane comme la plus experte et il n'y aurait pas de différences fondamentales entre le jugement d'un profane et celui d'un expert, le premier étant simplement plus spontané et «saturé d'expériences et d'intuitions personnelles» alors que le second s'est «décanté» par le biais d'une justification argumentée (Rochlitz, 1998, p. 168, cité par Uzel, 2004).

\subsection{Esthétique et Éthique}

La caractérisation kantienne du jugement de gout comme désintéressé et sans concept est l'un des points de départ du processus d'autonomisation du Beau et du Bien qui voit le jour au début du XIX ${ }^{\mathrm{e}}$ siècle et qui trouve son apogée dans les années 1960-1970 avec les approches formelles de la littérature. Parce que désintéressée et essentiellement contemplative, la relation esthétique s'attache désormais plutôt à la forme de la représentation et au plaisir qu'elle suscite qu'à sa mimesis, comprise comme la représentation d'actions ou d'évènements (TalonHugon, 2009; Sapiro, 2011). Selon Madame de Staël (1810) ${ }^{4}$, ce qui est beau peut éventuellement faire émerger des «sentiments généreux» et donc inciter à la vertu, mais «dès qu'on a pour objet de mettre en évidence un précepte de morale, la libre impression que produisent les chefs-d'œuvre de l'art est nécessairement détruite» (De Staël, 1814, citée par Sapiro, 2011, p. 14). Tout ce qui dans l'œuvre est de l'ordre de

3. Pensons par exemple aux blogs de lecture dans lesquels un lecteur propose une critique de tel livre, critique ensuite commentée, discutée par les internautes.

4. Comme l'indique Sapiro (2011), la théorie kantienne est largement relayée par Madame de Staël au début du XIX ${ }^{e}$ siècle alors que celle-ci n'est pas encore traduite en français (p. 14). 
l'utile ou de la morale la discrimine indéniablement, car n'est vraiment beau que ce qui ne sert à rien (Sapiro, 2011). En affirmant cette incompatibilité entre art et morale, les tenants de l'art pour l'art se démarquent ainsi catégoriquement de la doctrine classique fondée principalement sur l'Utile dulci d'Horace qui accordait dans son Art poétique ( $1^{\text {er }}$ siècle av. J.-C./1955) tous ses «suffrages» au poète «qui a mêlé l'utile à l'agréable, en donnant au lecteur du plaisir et de l'instruction» (vers 334340). Cette autonomisation entre esthétique et morale se retrouve également chez des théoriciens du $\mathrm{xx}^{\mathrm{e}}$ siècle tels que Genette et Schaeffer qui reprennent à leur compte l'approche kantienne du jugement de gout.

Pourtant depuis quelques années sous l'influence des cultural studies, des approches post-bourdieusiennes de l'art avec notamment les travaux de Nathalie Heinich, mais surtout face à certaines propositions artistiques et littéraires contemporaines, la question de la relation entre art et éthique refait surface. Peut-on réellement, lors de la réception d'un roman par exemple, dissocier les dimensions formelles des actions et des comportements des personnages? Selon Rabaté (2007), qui revient également à Kant pour souligner la dimension argumentative du jugement, il s'agit de formuler une vision étendue de la relation esthétique. Ce ne sont pas uniquement les jugements relatifs à la prétendue beauté d'une œuvre donnée qui la caractérisent, mais également des jugements d'ordre éthique ou «idéologique». Rabaté (2007) ne prône pas l'application de critères externes objectifs aux œuvres, mais souligne plutôt la nécessité d'articuler cette question des valeurs (esthétiques, éthiques) «aux modalités complexes de l'expression» des œuvres littéraires, en prenant en considération notamment leur «scénographie énonciative» (p. 16).

L'analyse de l'énonciation, de la polyphonie ou de la scénographie des textes littéraires permet de déterminer ce que Baroni (2007) appelle la responsabilité de l'auteur, la portée morale de certains écrits, mais également «leur prétention à fournir l'accès à une réalité ou à une vérité». Les approches récentes de la relation à l'œuvre littéraire diffèrent ainsi de l'approche classique.

Il n'est plus tant question de considérer l'art dans son efficace jonction entre le plaire et l'instruire, mais de prendre conscience que l'œuvre est le fruit d'une intention artistique, obéissant à une visée, à un dessein, en attente d'une validation publique. L'œuvre littéraire, comme l'affirme Maingueneau (2004), ne se destine pas à la contemplation, elle est une «énonciation tendue vers un co-énonciateur» qu'elle cherche à «mobiliser» afin de le faire «adhérer à un certain univers de sens» (p. 203). 
Cette validation publique, intersubjective, passe par le biais d'un débat démocratique entre sujets libres. Non pas production d'une preuve ou dispute, mais «débat ouvert entre égaux de droit» animés par la même volonté de convaincre (Rabaté, 2007, p. 24; Citton, 2007).

\section{3. Émotions : entre (dé)plaisir esthétique et passions}

On peut distinguer deux types de rapports émotionnels à l'œuvre littéraire. Le premier, qu'on peut désigner comme (dé)plaisir esthétique, est lié à la relation esthétique au texte littéraire. Il présuppose des catégories esthétiques qui, par l'expérience de l'œuvre littéraire, se confirment et se différencient. La reconnaissance de l'individualité de l'artiste propre à la modernité coïncide avec le développement et la reconnaissance d'une spécificité esthétique du plaisir (Lontrade, 2004). Kant, qui confère la primauté à l'expérience et à la relation que le sujet entretient avec une œuvre, défend que le plaisir est antérieur au jugement, sorte de «pré-juger» autonome qui «servir(ait) d'assise au jugement de gout» (Lontrade, 2004, p. 15). Chez des théoriciens comme Genette ou Schaeffer, le plaisir est également au centre de la relation aux œuvres. Pour Schaeffer, la conduite esthétique se caractérise invariablement par une activité cognitive et une réaction affective - l'attention portée à une œuvre étant régulée par le plaisir qu'elle engendre. La relation de (dé)plaisir peut ainsi être conçue comme immédiate et se manifestant sous forme de réactions spontanées à l'œuvre. Elle peut être qualifiée d'autotélique en ce sens qu'elle considère le plaisir comme une finalité en soi. Elle est pourtant, de fait, le résultat d'une longue expérience, le fruit d'une éducation.

Il nous semble que les émotions que font apparaitre les élèves dans leur relation au texte sont d'un autre ordre, réellement immédiates : affection, rejet, identification, «happage ${ }^{5} »$, surprise. Ces émotions ne sont pas filtrées - ou presque - par des catégories esthétiques. Elles seraient plutôt de l'ordre des passions, liées à l'effet de la mimesis sur le lecteur, c'est-à-dire à la manière dont les actions, les évènements ou les personnages d'une histoire l'affectent. Les travaux de l'équipe Lettres Langages et Arts apportent un éclairage intéressant quant à la place des affects dans l'appropriation d'une œuvre littéraire. En effet, selon Fourtanier et Langlade (2007), l'activité de fictionnalisation lectorale passe entre autres par une activation fantasmatique, c'est-à-dire la sol-

5. Nous reprenons ici le néologisme de Legallois et Poudat (2008). 
licitation par le texte de l'inconscient du lecteur, par exemple ses peurs, ses désirs, ses fantasmes, etc. Cette activation se traduit par l'attrait ou le rejet, la fascination ou le dégout vis-à-vis de certains éléments du texte, par exemple tels comportements d'un personnage, ou telle valeur induite par l'œuvre. Cette affectation n'est pas uniquement due à l'histoire en tant que telle, elle est induite également par la manière dont elle est racontée ${ }^{6}$.

\section{Opérationnalisation et méthodologie de la recherche}

\subsection{Le jugement comme unité d'analyse}

Notre objectif est de comprendre et d'analyser les différents types de relations co-construites en classe par les élèves et les enseignants vis-àvis du texte littéraire. En fonction du cadre théorique présenté ci-dessus et de nos données empiriques, nous avons construit une unité d'analyse, le «jugement», qui nous apparait comme révélateur de certaines relations au texte littéraire en classe. Il s'agit de portions de textes qui contiennent au minimum une caractérisation d'un objet textuel d'un point de vue esthétique, éthique ou émotionnel, dimensions définies en fonction de notre cadre théorique. Le repérage des «jugements» s'est donc opéré en fonction de trois critères : énonciation à propos d'un élément du texte; caractérisation de cet élément; dimension esthétique, éthique ou émotionnelle.

Le repérage a permis de produire une liste de jugements pour chaque texte. Pour analyser cette liste de jugements, nous avons élaboré une grille d'analyse en nous appuyant sur : 1) la théorie didactique, notamment un article de Dufays (2000) dans lequel il répertorie les différentes valeurs pouvant être convoquées lors de la lecture de textes littéraires en classe ; 2) certaines approches en analyse du discours, notamment un article de Galatanu (2003) qui propose un tableau récapitulatif des valeurs modales; 3 ) certaines analyses empiriques réalisées en dehors de l'école et portant sur des jugements d'œuvres littéraires, notamment celles de Legallois et Poudat (2008) qui reprennent les catégorisations de Dufays tout en les modifiant en fonction de leurs données. Sur cette

6. Voir à ce propos les travaux de Wayne Booth (1961/1988) qui développent ce qu'il nomme une rhétorique de la fiction, travaux repris et développés notamment par Ricœur (1985) et Baroni (2006). 
base, nous avons défini une série de sous-catégories pour chacune des trois dimensions fondamentales (esthétique, éthique et émotions) dont on trouve une présentation en annexe. Cette grille a permis de classer l'ensemble des jugements, chaque jugement pouvant d'ailleurs comprendre plusieurs catégories ou dimensions.

\subsection{Particularité méthodologique de la recherche}

Notre travail s'appuie sur un corpus issu d'un dispositif semi-expérimental de recueil de données. Il s'inscrit dans une vaste recherche subventionnée par le FNS menée par l'équipe GRAFE consacrée à l'enseignement de la lecture de textes littéraires dans des écoles genevoises de différents niveaux ${ }^{7}$. Afin de mieux comprendre comment s'instaurent des relations esthétiques, éthiques et émotionnelles en classe ainsi que les évaluations qui en découlent, mais également comment elles participent à la formulation d'interprétations, nous analysons comment deux textes contrastés - l'un fortement didactisé et appartenant à la vulgate scolaire (Le loup et l'agneau de Jean de La Fontaine), l'autre issu de l'extrême contemporain et n'ayant fait l'objet d'aucune didactisation (La négresse et le chef des avalanches de Jean-Marc Lovay) - sont abordés dans des classes de primaire, de secondaire I et de secondaire II. Nous cherchons à comparer ainsi l'impact du texte et du niveau scolaire sur l'élaboration et la formulation de jugements de type esthétique, éthique et émotionnel. Nous nous posons plus particulièrement les questions suivantes : les relations aux textes et les jugements qui en témoignent sont-ils les mêmes lorsqu'il s'agit d'aborder une œuvre du patrimoine scolaire ou une œuvre contemporaine non encore validée par l'institution scolaire? Les rôles joués par les enseignants et les élèves dans le processus d'évaluation sont-ils identiques pour les deux textes? Existe-t-il une variation en fonction des niveaux scolaires? Dans quelle mesure la formulation de jugements, l'engagement émotionnel du sujet lecteur, sa relation de (dé)plaisir ou son rapport éthique vis-à-vis du texte participent-ils d'un processus interprétatif plus large propre à la lecture littéraire?

7. Fonds national suisse de la recherche scientifique $n^{\circ} 100013 \_129797$ : «La lecture de textes littéraires au fil des niveaux scolaires. Analyse comparative des objets enseignés ». Recherche sous la direction de B. Schneuwly et C. Ronveaux. 
Dans la présente contribution, nous proposons une première analyse des jugements effectués par les élèves et les enseignants concernant les deux textes. Nous procédons en deux temps. D'abord, nous analysons les jugements du point de vue quantitatif, pour présenter au lecteur une image des grandes tendances qu'on peut observer. Ensuite, en fonction de cette analyse, nous étudions plus en détail et qualitativement deux phénomènes particulièrement significatifs de jugements des textes.

\section{Analyse quantitative : la progression n'est pas toujours linéaire}

\subsection{De plus en plus de jugements... une progression linéaire}

Le tableau 1 montre le nombre de séquences sur lesquelles porte notre analyse quantitative.

\begin{tabular}{lccc}
\hline & LAF & LOV & Total \\
\hline Primaire & 10 & 10 & 20 \\
Secondaire I & 9 & 9 & 18 \\
Secondaire II & 8 & 8 & 16 \\
\hline Total & 28 & 27 & 54 \\
\hline
\end{tabular}

Tableau 1. - Nombre de séquences analysées ${ }^{8}$.

567 jugements ont été repérés dans ces 54 séquences. Ceci donne une moyenne de 10 jugements par séquence. L'analyse par niveau montre une immense différence des moyennes : elle double du primaire au secondaire I et encore une fois du secondaire I au secondaire II.

8. Pour cette analyse quantitative, nous avons en effet éliminé les séquences 2.8 du secondaire I qui totalisait à elles seules autant de jugements que toutes les autres réunies. Quant au secondaire II, pour des raisons techniques, nous n'avons pas encore pu analyser les séquences 3.9 et 3.10 . 


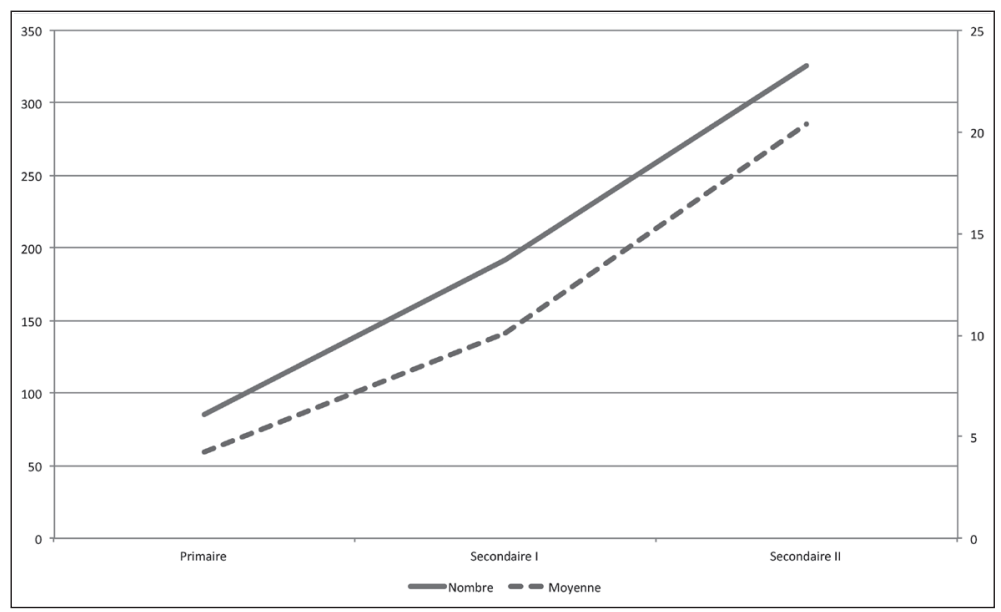

Figure 1. - Nombre de jugements en valeur absolue et en moyenne par séquences en fonction des niveaux.

Cette évolution n'est pas du tout liée à la longueur des séquences qui, elle, reste constante : en moyenne deux séquences par classe, une par texte abordé. Ces chiffres marquent donc un déplacement immense des discours. D'autres analyses nous montrent que ce déplacement résulte du fait que le travail sur la compréhension du texte, sur le vocabulaire, et même sur l'élaboration du contexte du texte, prend une place infiniment plus grande au primaire, encore partiellement au secondaire I et qu'elle disparait quasiment au secondaire II. L'activité de travail sur le texte, dont les jugements sont une manifestation particulièrement significative, se développe puissamment au cours de la progression scolaire.

\subsection{Le texte n'a apparemment pas d'effet : les élèves parlent de moins en moins}

Une analyse plus fine permet d'observer deux autres phénomènes.

L'une de nos questions portait sur l'effet du texte. Or, au niveau purement quantitatif, cet effet ne se manifeste pas. 


\begin{tabular}{lcccc}
\hline & \multicolumn{2}{c}{ Nombre } & \multicolumn{2}{c}{ Moyenne } \\
& LAF & LOV & LAF & LOV \\
\hline Primaire & 31 & 54 & 1,6 & 2,7 \\
Secondaire I & 97 & 95 & 5,1 & 5,0 \\
Secondaire II & 160 & 166 & 10,0 & 10,4 \\
\hline Total & 288 & 315 & 5,2 & 5,7 \\
\hline
\end{tabular}

Tableau 2. - Nombre de jugements et moyenne en fonction de deux textes contrastés et des niveaux.

Le tableau 2 montre en effet que le nombre de jugements, contrairement aux attentes, ne dépend pas du texte travaillé, sauf au primaire où le texte de Lovay en suscite un nombre significativement plus élevé. Tout se passe comme si, à ce niveau, l'étrangeté du texte impliquait, ou permettait, une certaine distance même au niveau primaire.

Les jugements sont prononcés par des élèves ou par des enseignants. Le tableau 3 fait l'analyse en fonction de ce facteur.

\begin{tabular}{lccccc}
\hline & \multicolumn{2}{c}{ Nombre } & \multicolumn{2}{c}{ Moyenne } & \\
& Enseignants & Élèves & Enseignants & Élèves & $\%$ \\
\hline Primaire Total & 33 & 52 & 1,7 & 2,6 & 63,5 \\
Secondaire I Total & 73 & 119 & 3,8 & 6,3 & 61,3 \\
Secondaire II Total & 159 & 167 & 9,9 & 10,4 & 95,2 \\
\hline Total & 265 & 338 & 4,8 & 6,1 & 78,4 \\
\hline
\end{tabular}

Tableau 3. - Nombre et moyenne de jugements en fonction des niveaux et des locuteurs.

Les tendances sont claires et surprenantes, du moins à première vue : aussi bien au primaire qu'au secondaire I, les élèves énoncent nettement plus de jugements qu'au secondaire II où le rapport s'équilibre. Serait-ce ici un effet de ce que décrit Bautier (2008) :

Pour plusieurs raisons, tout se passe depuis plus d'une dizaine d'années comme si les élèves, après avoir été durant des décennies des élèves «écoutants», écoutant la parole transmissive de l'enseignant, étaient devenus, ou devaient devenir, des êtres scolaires de parole et d'énonciation à qui il est nécessaire d'accorder une place dans la classe. (p. 3)

Mais alors, il semblerait bien que, contrairement à ce qu'elle observe, le phénomène serait moins marqué au secondaire II. L'analyse plus 
détaillée des discours permettra de mieux comprendre le phénomène et surtout de voir dans quelle mesure le langage utilisé et ce qui y est dit participe d'une sorte d'indistinction entre parler quotidien et langage spécialisé.

\subsection{L'effet texte retrouvé: progression attendue pour La Fontaine, inattendue pour Lovay}

Passons maintenant à l'analyse des jugements. Comment se répartissentils sur les trois dimensions principales que nous avons distinguées dans la «relation» à la littérature, qui se manifeste à travers les jugements esthétiques, éthiques et émotionnels ? Pour ce faire, nous allons raisonner non plus en termes de nombre, mais présenter les parts de chaque dimension dans l'ensemble des jugements. Cette manière de faire permet de faire abstraction des nombres, très inégaux en fonction des niveaux comme nous l'avons vu. Définir des parts permet d'obtenir une sorte de description quantitative des jugements émis. Rappelons qu'un jugement peut simultanément porter sur deux dimensions.

\begin{tabular}{lcccc}
\hline & Esthétique & Éthique & Émotion & Total \\
\hline LAFPrimaire & 30,8 & 51,3 & 17,9 & 100 \\
LAFSecondaire I & 38,2 & 48,1 & 13,7 & 100 \\
LAFSecondaire II & 60,1 & 37,3 & 2,6 & 100 \\
\hline LOVprimaire & 51,8 & 25,9 & 22,4 & 100 \\
LOVSecondaire I & 47,6 & 39,7 & 12,7 & 100 \\
LOVSecondaire II & 55,8 & 37,5 & 6,8 & 100 \\
\hline Primaire Total & 45,2 & 33,9 & 21,0 & 100 \\
Secondaire I Total & 42,8 & 44,0 & 13,2 & 100 \\
Secondaire II Total & 58,0 & 37,4 & 4,6 & 100 \\
\hline LAF Total & 51,0 & 41,7 & 7,3 & 100 \\
LOV Total & 52,8 & 35,9 & 11,3 & 100 \\
\hline Total & 51,9 & 38,8 & 9,3 & 100 \\
\hline
\end{tabular}

Tableau 4. - Pourcentage de jugements en fonction de trois dimensions, des deux textes et des niveaux scolaires.

Le tableau montre (ligne «Total»), que la dimension «esthétique» est la plus fréquente, textes et niveaux confondus. Cette distribution des jugements ne diffère pas fondamentalement en fonction des textes 
(«LAF Total» versus «LOV Total»), contrairement à nos attentes. On observe toutefois que le texte de Lovay suscite tendanciellement plus de jugements d'émotion et moins de jugements éthiques, tendance cependant peu marquée.

Regardons maintenant la distribution des trois dimensions en fonction du niveau scolaire (lignes «Primaires, Secondaire I et Secondaire II Total»). Les tendances sont relativement complexes (voir aussi les fig. 2 et 3). On constate d'abord une même tendance pour tous les textes concernant les jugements d'émotion : leur part baisse régulièrement du niveau du primaire vers le niveau du secondaire. Le phénomène souvent noté d'une objectivation du jugement s'observe ici de manière particulièrement claire. L'émotion directement exprimée se transforme en jugement esthétique ou éthique; c'est du moins ce qu'on peut observer dans nos données.

Les tendances sont par contre moins régulières en ce qui concerne les deux autres dimensions. Si l'on constate globalement une augmentation de la part des jugements esthétiques entre le primaire et le secondaire II, au secondaire I, on note même une légère baisse. Inversement, la part des jugements éthiques augmente de manière assez importante au secondaire I.

Les figures 2 et 3 montrent l'évolution en fonction des textes de La Fontaine et de Lovay.

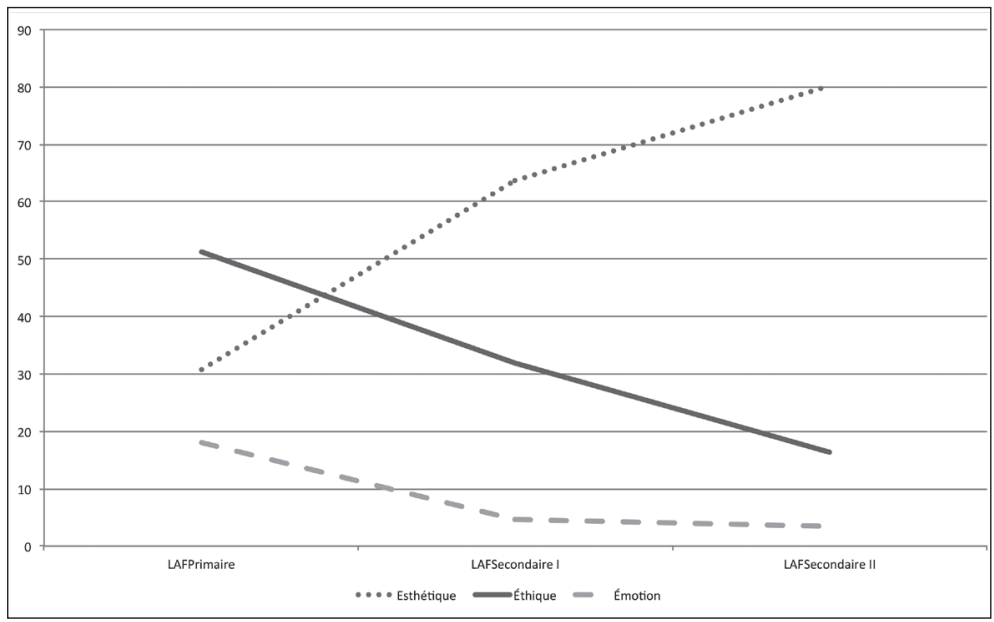

Figure 2. - Évolution de la part des jugements en fonction du niveau scolaire et des dimensions dans le texte de La Fontaine. 
Dans La Fontaine, on observe une évolution qu'on pourrait dire «régulière»: la part des dimensions «émotions» et «éthiques» baisse au profit de la dimension «esthétique». Tout se passe comme si le texte devenait de plus en plus l'objet d'un travail scolaire classique, avec en point de mire les dimensions formelles : style, intrigue, signifiants notamment.

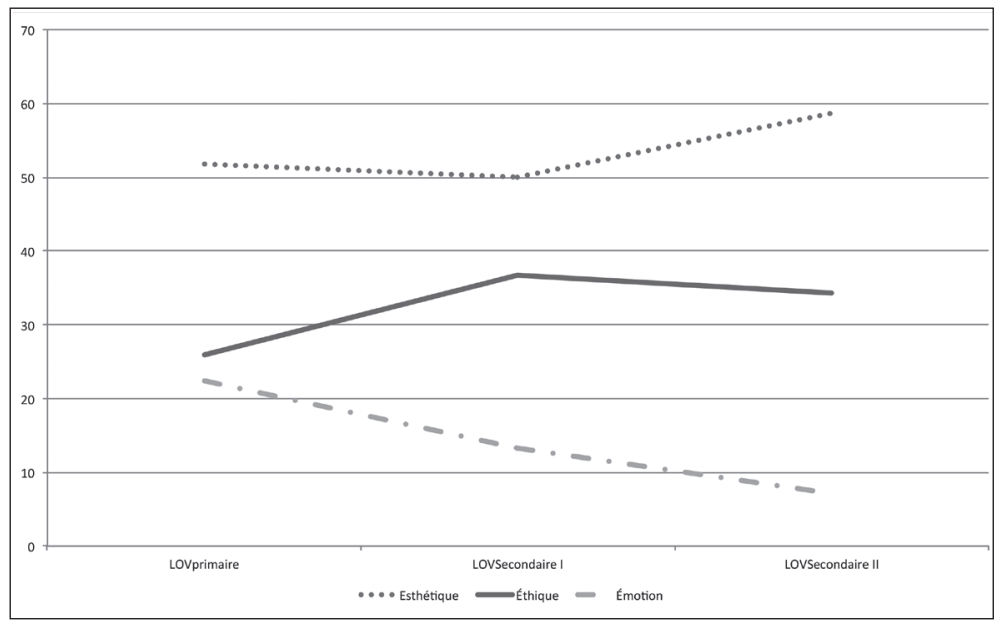

Figure 3. - Évolution de la part des jugements en fonction du niveau scolaire et des dimensions dans le texte de Lovay.

Il en va autrement pour le texte de Lovay qui visiblement perturbe l'ordonnancement régulier de l'évolution (voir fig. 3). En effet, aussi bien la part des jugements concernant les dimensions esthétiques que celle concernant les dimensions éthiques augmentent globalement, au détriment toujours de la dimension «émotion». Plus surprenant encore : la part des jugements esthétiques diminue au secondaire I comparé au primaire, pour atteindre un niveau plus élevé au secondaire II.

Comment expliquer ce phénomène? La première observation qu'on peut faire : les jugements éthiques concernant le texte de Lovay émanent significativement plus souvent des élèves que des enseignants pour le degré secondaire I et secondaire II, comme le montre le tableau 5 : 


\begin{tabular}{lcccc}
\hline & \multicolumn{2}{c}{ Esthétique } & \multicolumn{2}{c}{ Éthique } \\
& Enseignant & Élève & Enseignant & Élève \\
\hline LOVprimaire & 20,0 & 31,8 & 10,6 & 15,3 \\
LOVSecondaire I & 26,7 & 23,3 & 12,5 & 24,2 \\
LOVSecondaire II & 32,2 & 26,4 & 10,9 & 23,4 \\
\hline
\end{tabular}

Tableau 5. - Part de jugements esthétique et éthique en fonction du niveau et des locuteurs enseignant et élève.

On observe que pour le jugement éthique, contrairement au jugement esthétique, la part des élèves augmente significativement. Elle constitue le double de ceux émanant des enseignants. Autrement dit : ce sont les élèves qui réagissent à ce qu'ils considèrent comme contenu éthique du texte de Lovay, et plus particulièrement à ce qu'évoque le mot «négresse » et le rôle qu'ils croient que joue ce personnage dans le texte. Nos analyses qualitatives permettront de mieux comprendre ce phénomène.

\section{Analyses qualitatives}

Nous allons à présent analyser plus en détail un certain nombre de jugements afin de prolonger et développer nos premières analyses. Nous nous penchons tout particulièrement sur les jugements formulés sur les personnages de la fable au fil des niveaux scolaires. Puis, dans un deuxième temps, nous nous intéressons aux jugements portant sur l'emploi du terme de «négresse» par Lovay ainsi que sur le rôle joué dans la nouvelle par ce personnage singulier.

\section{1. À propos des personnages de la fable}

Concernant le texte de La Fontaine, nos analyses quantitatives ont montré une baisse régulière, du primaire au secondaire II, de la part des dimensions «Émotion» et «Éthique». Nous aimerions dans un premier temps étudier cette évolution à travers l'analyse qualitative de certains jugements formulés à propos du loup et de l'agneau. Lors de la construction de notre grille d'analyse des jugements, nous avons été amenés à distinguer deux types de jugements sur les personnages. Le premier type, rattaché à la catégorie générale «Éthique», considère le personnage comme dégagé de ses caractéristiques textuelles. Par l'intermédiaire d'un phénomène d'«effet de vie» (Jouve, 2001, p. 108), les 
personnages de la fable sont appréhendés comme des êtres vivants. Pour cette raison, nous avons intitulé cette sous-catégorie «personne». Ce type de jugement se retrouve essentiellement au primaire. Les élèves, de manière systématique, appréhendent le loup et l'agneau comme des personnes en chair et en os, sans prendre en considération le fait que ce sont des animaux. Ce qui est jugé, évalué, sont les comportements des deux protagonistes. En voici deux exemples :

El : le loup est idiot mais fort des muscles. (LAF_1_4/44) ${ }^{9}$

Ou encore :

El : c'est injuste il devrait le manger mais lui il a rien fait, il devrait plutôt manger celui qui fait quelque chose. (LAF_1_8/81)

Ces jugements d'ordre éthique portés sur les personnages se couplent fréquemment d'un investissement affectif et identificatoire spontané de la part des élèves (couplage des catégories «Éthique» et «Émotion» avec les sous-catégories «personne» et «identification») :

El : moi je me serai battu. (LAF_1_4 / 40)

Il arrive à quelques reprises qu'un élève ne semble pas perturbé par la «morale» ambigüe de la fable. Ce sont alors les enseignants qui insistent sur l'injustice commise envers l'agneau :

Ens : qu'est-ce que vous pensez de ça (...) par exemple ton frère se comporte mal avec quelqu'un et ce quelqu'un vient se venger sur toi

(...) tu trouves ça normal $\uparrow$

$\mathrm{El}:$ non

Ens : donc là ce n'est pas normal ce qu'il dit le loup. (LAF_1_7/22-23)

Au travers de la convocation de la vie quotidienne de l'élève, l'enseignant entend certainement impliquer ce dernier dans la fable afin qu'il comprenne, intimement, l'injustice qu'elle met en scène. Se dessine ici, en filigrane, les à priori esthétiques du milieu scolaire quant aux œuvres littéraires patrimoniales. Une belle œuvre n'est-elle pas celle qui met en avant de bonnes valeurs morales? Toutefois, cette convoca-

9. Comme plus haut, LAF désigne le texte de La Fontaine. 1_4 : le premier chiffre donne l'information sur le niveau (1: primaire; 2 : secondaire I; 3 : secondaire II). Quant au chiffre qui suit, il désigne celui de la leçon. Le dernier réfère au jugement. 
tion de la vie quotidienne se fait au détriment du texte lui-même et de sa forme qui deviennent un implicite non thématisé (Bautier, 2008).

Le second type de jugements relatifs aux personnages se rattache, quant à lui, à la catégorie générale «Esthétique». Il reflète une conception des protagonistes comme un construit textuel et s'articule fréquemment à l'analyse de la scénographie énonciative de la fable. C'est pour cette raison précisément que nous avons nommé cette sous-catégorie «personnage». Encore peu présent au secondaire I, ce type de jugement se retrouve essentiellement au secondaire II, plus particulièrement dans les séquences sur La Fontaine. La dimension identificatoire et affective relevée au primaire et encore très présente au secondaire I, devient plus rare, proportionnellement, au secondaire II. Les élèves considèrent d'emblée les protagonistes de la fable comme des construits, des symboles.

El : (le loup) se [met] en victime et les hommes [sont] contre lui et ben dans la nature c'est pas comme ça que ça se passe. (LAF_3_4 / 113)

Les jugements deviennent par ailleurs plus difficilement séparables d'interprétations :

El : l'agneau il a une bonne rhétorique et il sait quand même répondre on ne pourrait pas le comparer au peuple qui savait pas parler lire écrire et tout ça. (LAF_3_4 / 119)

Une chose mérite également d'être soulignée : les élèves, tout comme les enseignants, reviennent très fréquemment au texte, le citent, pour appuyer tel ou tel jugement :

$\mathrm{El}:$ : il cherche aventure» c'est quelque chose de négatif. (LAF_3_4 /

Les enseignants, quant à eux, encouragent les élèves à s'engager dans l'analyse de l'énonciation, en cherchant par exemple à déceler le rôle du narrateur dans la présentation des personnages :

Ens : le narrateur désigne le loup par «bête cruelle» et par «animal plein de rage» autrement dit il apporte une connotation péjorative. (LAF_3_8 / 299)

Ou encore :

Ens : et dans le discours du loup on retrouve exactement la même chose les motifs premiers affichés ne sont pas les vrais motifs qui existent on retrouve la même structure du masque. (LAF_3_3 / 73) 
Ainsi, au primaire, les jugements des élèves montrent leur investissement dans le texte et les processus d'identification vis-à-vis des personnages. Les élèves considèrent l'agneau et le loup précisément comme des «personnes». Les jugements sont donc placés sous le signe de l'éthique et de l'émotion. Au fil des niveaux, ces jugements «saturés d'expériences personnelles», pour reprendre l'expression de Rochlitz, se décantent. La dimension émotionnelle disparait au profit des outils d'analyse acquis au fil de la scolarité. Il devient d'ailleurs parfois extrêmement difficile de distinguer les jugements d'interprétations. On passe d'une relation au texte littéraire comme modèle de bonne conduite, au texte comme œuvre d'art langagière présupposant un rapport particulier, un rapport esthétique.

\section{2. "La négresse et le chef des avalanches", un texte raciste?}

Les analyses quantitatives ont montré que la disparition régulière des jugements éthiques et émotionnels au profit de jugements esthétiques ne se vérifie pas pour le texte de Lovay. En effet, les jugements éthiques sont particulièrement nombreux au secondaire I comparativement au primaire et au secondaire II. Nous aimerions illustrer cette «anomalie» en analysant les jugements formulés par rapport au terme «négresse». Au primaire, de manière très intéressante, les considérations portant sur ce terme, ou sur le personnage en tant que tel, ne sont que rarement couplées avec une dimension émotionnelle. Les élèves s'interrogent sur le rôle et la signification de la négresse dans l'histoire et emploient un certain nombre de modalisateurs afin de nuancer quelque peu leurs propos. En voici deux exemples significatifs :

El : j'ai trouvé bizarre parce qu'en même temps raciste enfin je sais pas c'est méchant de dire que parce qu'on pose une noire sur la cage les avalanches ne vont pas arriver. (LOV_1_3/15)

$\mathrm{Ou}$ :

$\mathrm{El}$ : c'est pas très sympa de dire euh une avalanche tellement qu'elle est blanche et la négresse tellement elle est noire qu'elles vont avoir peur. (LOV_1_10 / 86)

Dans ces deux extraits, les élèves semblent avancer prudemment l'idée de racisme. Probablement l'étrangeté du texte, mais également son caractère provocateur (l'emploi du terme négresse dans le titre et le rôle de la négresse dans l'histoire, entre objet et divinité) interpelle les élèves qui n'ont peut-être pas vraiment l'habitude d'être confrontés à ce genre 
de textes littéraires. C'est du moins ce que laisse penser la citation suivante :

El : j'ai trouvé ça bête mais intelligent parce que ça montre que les blancs ont peur des noirs donc ça montre que nous on a peur de la différence mais aussi «négresse» des mots comme ça je trouve ça un peu bête faut justement pas qu'on les apprenne et on nous les met dans un livre. (LOV_1_2/5)

Au secondaire I, les dimensions éthiques sont particulièrement importantes. L'analyse quantitative montre également que ces jugements sont principalement formulés par les élèves. Les réactions de ces derniers, en comparaison avec celles des élèves de primaires, sont beaucoup plus virulentes. La formulation est très différente, beaucoup plus directe, affirmative. Les modalisateurs sont peu présents :

El : voilà on les traite comme des chiens c'est du racisme. (LOV_2_3/15)

$\mathrm{Ou}$ :

$\mathrm{El}$ : c'est un gros raciste. (LOV_2_3/22)

$\mathrm{Ou}$ :

El : et euh bah voilà et puis ils l'exploitent après en fait c'est du racisme c'est de l'exploitation. (LOV_2_5/ 51)

Les enseignants face à ces réactions tentent de ramener les élèves au texte, de faire reformuler l'intrigue, de distinguer auteur et narrateur, etc. Voici une interaction particulièrement intéressante :

Kao : c'est un raciste $\uparrow$

Ens : c'est un raciste $\uparrow$ qui est raciste $\uparrow$

Kao : l'auteur parce que c'est comme s'il disait quand il y la négresse qui arrive il y a tout le monde qui s'enfuit $\downarrow$

Ens : alors $\downarrow$ il y a une idée de racisme $\downarrow$ est-ce qu'on peut taxer l'auteur de raciste $\uparrow$

$\mathrm{El}(\mathrm{s}):$ non

Ens : parce qu'il a utilisé le mot <négresse > $\downarrow$

$\mathrm{El}(\mathrm{s})$ : non

Ens : est-ce qui xxx alors possible mais il aurait aussi pu faire autre chose

Mic : ben en fait il essaie de faire passer un message

Ens : voilà voilà $\uparrow$

(LOV_2_2 / lignes 212-222) 
Ces réactions virulentes ont une nette tendance à disparaitre au secondaire II. Le caractère problématique du terme «négresse» n'est pas occulté, mais il s'inscrit dans une démarche interprétative générale. Les modalisations (en italique) sont à nouveaux très présentes :

El : monsieur est-ce que ça peut être un texte raciste $\uparrow\left(L O V \_3 \_3 / 94\right)$ Ou :

$\mathrm{El}:$ «sinon ils équiperont de négresses» en fait la négresse c'est un objet si c'est péjoratif le sens premier de négresse en fait enfin c'est trop tôt pour le dire. (LOV_3_3 / 100)

Ou :

El : moi j'ai mis que c'était aussi un texte qui était peut-être raciste même si y a des termes racistes comme «négresse» dedans que y avait une sorte de bataille entre le blanc et le noir mais qu'ils essayent de vaincre le racisme. (LOV_3_4 / 175)

Précisons que ces trois extraits se trouvent en début de leçon. Les enseignants demandent aux élèves ce qu'ils ont pensé du texte après la lecture de ce dernier. La récurrence du terme «peut-être» ouvre la porte au contre-argument, laisse la place à la discussion avec l'enseignant ou avec les pairs. Ressort également de ces extraits une certaine conscience du temps. Comme l'indique l'autocommentaire ou autorégulation «il est trop tôt pour le dire», les élèves savent que le temps de la leçon permettra le déroulement cette fois-ci herméneutique de l'analyse. Les élèves d'ailleurs, au fur et à mesure que la leçon avance, délaissent l'impression de racisme pour se concentrer sur la recherche des sens possibles :

El : mais enfin de façon générale quand ils disent qu'elle va nous sauver des avalanches etcetera à la fin (cite) <elle va nous montrer le froid je la poserai au-dessus de la cage toute noire et les avalanches auront peur> c'est vraiment un symbole comme si elle allait sauver la civilisation. (LOV_3_8 / lignes 735-739)

\section{Conclusion}

Ces analyses tant quantitatives que qualitatives mettent en avant la pertinence de notre unité d'analyse. En effet, les données empiriques GRAFELIT nous montrent que toute relation à l'œuvre littéraire en classe, qu'elle soit esthétique, éthique et/ou émotionnelle est marquée par le sceau du jugement. Ces jugements portés sur les textes par les 
élèves et les enseignants augmentent à mesure que la lecture du texte se fait interprétative. Le jugement serait donc indissociable de l'interprétation. Par ailleurs, notre analyse des jugements met en lumière la transformation, au fil des niveaux scolaires, de l'activité «lecture de textes littéraires ». La disparition progressive, pour les deux textes, de jugements émotionnels - c'est-à-dire la disparition de réactions affectives spontanées ou de processus identificatoires au profit d'une approche plus distanciée, esthétique, propre au secondaire II - rend compte d'un phénomène d'objectivation concomitante à l'acquisition d'un certain nombre d'outils propres à l'analyse de texte. Cette transformation est visible jusque dans le langage même des élèves qui, dès lors qu'ils sont au secondaire II, emploient un plus grand nombre de modalisateurs et manient plus aisément le vocabulaire de l'analyse. La nature des relations à l'œuvre littéraire, particulièrement en ce qui concerne le texte de La Fontaine, évolue ainsi en fonction du niveau scolaire, passant des relations plutôt éthiques et émotionnelles à une relation essentiellement esthétique, ou, plus précisément, à l'imbrication de dimensions éthiques «aux modalités [plus formelles] de [leur] expression» (Rabaté, 2007, p. 16). Si les discours sur les deux textes ne se distinguent pas au niveau du nombre de jugements, leur nature quant à elle diffère. Nous avons vu que le texte de Lovay, par son étrangeté, ses spécificités littéraires, conduit des élèves du primaire à une relation plus distanciée. Face aux indéterminations du texte, on s'interroge sur les possibles significations ainsi que sur les intentions de l'auteur. Cette distanciation s'affaiblit pourtant au secondaire I avec des élèves dont les préoccupations principales sont essentiellement d'ordre éthique, préoccupations aiguillées par la dimension prétendument raciste de la nouvelle de Lovay. Ce texte de l'extrême contemporain résiste aux modes d'analyse habituellement mis en œuvre, cela jusqu'au secondaire II. Nos futures analyses montreront plus en détail les outils que mettent en œuvre les enseignants et élèves dans leur travail sur ces deux textes contrastés.

\section{RÉFÉRENCES BIBLIOGRAPHIQUES}

BAUTIER, Élisabeth. (2008). Ambitions et paradoxes des pratiques langagières scolaires : constructions au quotidien des inégalités sociales d'apprentissage. Communication présentée au colloque «Ce que l'école fait aux individus», CENS \& CREN. Disponible en ligne sur <http:// www.cens.univ-nantes.fr/71693378/0/fiche pagelibre/> (consulté le 24 juillet 2013). 
Bоотн, Wayne-Charles. (1961). The Rhetoric of Fiction. Chicago : University of Chicago Press.

Bоoтн, Wayne-Charles. (1988). The Company We Keep. An Ethics of Fiction. Los Angeles : University of California Press.

BARoni, Raphaël. (2007). La tension narrative. Paris : Seuil.

Baroni, Raphaël. (2006). Passion et narration. Protée, 34(2-3), 163-175. Disponible en ligne sur <www.erudit.org/revue/pr/2006/v34/n2-3/014 274ar.html> (consulté le 24 juillet 2013).

Citton, Yves. (2007). Lire, interpréter, actualiser. Pourquoi les études littéraires? Paris : Éditions Amsterdam.

Dufays, Jean-Louis. (2000). Lire, c'est aussi évaluer. Autopsie des modes de jugement à l'œuvre dans diverses situations de lecture. Étude de linguistique appliquée, 119, 227-290.

Galatanu, Olga. (2003). La construction discursive des valeurs. Dans J.-M. Barbier (dir.), Valeurs et activités professionnelles (p. 87-115). Paris : L'Harmattan.

Genette, Gérard. (1997). L'œuvre de l'art (t. 2 : La relation esthétique). Paris : Seuil.

Horace (1 ${ }^{\text {er }}$ siècle av. J.-C./1955). Épître aux Pisons. Dans Épîtres. Paris : Les Belles Lettres.

Jouve, Vincent. (2001). L'effet-personnage dans le roman. Paris : Presses universitaires de France.

Kant, Emmanuel. (1790/2011). Le jugement esthétique (textes choisis par F. Khodoss). Paris : Presses universitaires de France.

Langlade, Gérard \& Fourtanier, Marie-Josée. (2007). «La question du sujet lecteur en didactique de la lecture littéraire». Dans E. Falardeau, C. Fischer, C. Simard \& N. Sorin, La didactique du français. Les voies actuelles de la recherche. Québec: Presse de l'Université Laval.

Legallois, Dominique \& Poudat, Céline. (2008). Comment parler des livres que l'on a lus? Discours et axiologie des avis des internautes. Semen, 26. Disponible en ligne sur <http://semen.revues.org/8444> (consulté le 25 juillet 2013).

Lontrade, Agnès. (2004). Le plaisir esthétique. Naissance d'une notion. Paris : L'Harmattan.

Lovay, Jean-Marc. (1996). La négresse et le chef des avalanches. Genève : Zoé.

Maingueneau, Dominique. (2004). Le discours littéraire. Paratopie et scène d'énonciation. Paris : Armand Colin.

Rabaté, Dominique. (2007). La valeur comme question. Modernités, 25, 9-25.

Ricenur, Paul. (1985). Temps et récit (t. 3 : Le temps raconté). Paris : Seuil. 
Rochlitz, Rainer. (1998). L'art au banc d'essai. Esthétique et critique. Paris : Gallimard.

SAPIRo, Gisèle. (2010). Aux origines de la modernité littéraire : la dissociation du Beau, du Vrai et du Bien. Nouvelle revue de l'esthétique, 6 , 13-23. Disponible en ligne sur <www.cairn.info/revue-nouvelle-revued-esthetique-2010-2-page-13.htm> (consulté le 25 juillet 2013).

SCHAEFFER, Jean-Marie. (1996). Les célibataires de l'art. Pour une esthétique sans mythe. Paris : Gallimard.

Schneuwly, Bernard \& Dolz, Joaquim. (2009). Des objets enseignés en classe de français. Rennes: Presses universitaires de Rennes.

Talon-Hugon, Carole. (2009). Morales de l'art. Paris : Presses universitaires de France.

Uzel, Jean-Philippe. (2004). Rainer Rochlitz et l'ambivalence du jugement esthétique. Revue canadienne de l'esthétique, 9. Disponible sur <www. uqtr.uquebec.ca/AE/Vol_9/roch/Uzel.htm> (consulté le 24 juillet 2013).

Vouillou, Bernard. (2011). Par-delà le principe de plaisir esthétique. Aisthesis, 3, 23-37. Disponible en ligne sur <www.aisthesisonline.it/2011/ 1-2011/par-dela-le-principe-de-plaisir-esthetique/> (consulté le 24 juillet 2013). 


\section{ANNEXE}

\section{Grille d'analyse «jugements»}

\begin{tabular}{|l|l|l|}
\hline Catégories & Sous-catégories & Descriptions \\
\hline Éthique & morale & $\begin{array}{l}\text { Rapport à des normes } \\
\text { extérieures }\end{array}$ \\
\hline Esthétique & personne & Comportements et actions \\
\hline & intrigue & $\begin{array}{l}\text { Construction de l'intrigue, } \\
\text { clarté/complication }\end{array}$ \\
\hline & scénographie énonciative & $\begin{array}{l}\text { Degré d'implication du } \\
\text { locuteur }\end{array}$ \\
\hline & personnage & $\begin{array}{l}\text { Construction discursive, } \\
\text { inscription dans l'énoncia- } \\
\text { tion, dans l'intrigue, etc. }\end{array}$ \\
\hline & auteur & Intention, légitimation, etc. \\
\hline Émotion & intertexte & Rapport au canon littéraire \\
\hline & polysémie & $\begin{array}{l}\text { Rapport à d'autres textes } \\
\text { littéraires, comparaison }\end{array}$ \\
\hline & imagination & $\begin{array}{l}\text { Plusieurs pistes } \\
\text { interprétatives }\end{array}$ \\
\hline & style & $\begin{array}{l}\text { Travail sur la forme, la } \\
\text { langue, le lexique }\end{array}$ \\
\hline & signifiant & Compréhension \\
\hline & affectation & Effet psychologique \\
\hline & Effet psychologique \\
\hline & Réaction transférentielle \\
\hline & Effet psychologique \\
\hline & Effet psychologique \\
\hline & idenologique \\
\hline
\end{tabular}

